\title{
Gene Transfer of Connexin43 into Skeletal Muscle
}

\author{
HANS REINECKE, ELINA MINAMI, JITKA I. VIRAG, and CHARLES E. MURRY
}

\begin{abstract}
Cellular cardiomyoplasty using skeletal myoblasts may be beneficial for infarct repair. One drawback to skeletal muscle cells is their lack of gap junction expression after differentiation, thus preventing electrical coupling to host cardiomyocytes. We sought to overexpress the gap junction protein connexin43 (Cx43) in differentiated skeletal myotubes, using retroviral, adenoviral, and plasmid-mediated gene transfer. All strategies resulted in overexpression of $\mathrm{Cx} 43$ in cultured myotubes, but expression of $\mathrm{Cx} 43$ from constitutive viral promoters caused significant death upon differentiation. Dye transfer studies showed that surviving myotubes contained functional gap junctions, however. Retrovirally transfected myoblasts did not express $\mathrm{Cx} 43$ after grafting into the heart, possibly due to promoter silencing. Adenovirally transfected myoblasts expressed abundant Cx43 after forming myotubes in cardiac grafts, but grafts showed signs of injury at 1 week and had died by 2 weeks. Interestingly, transfection of already differentiated myotubes with adenoviral Cx43 was nontoxic, implying a window of vulnerability during differentiation. To test this hypothesis, $\mathrm{Cx} 43$ was expressed from the muscle creatine kinase (MCK) promoter, which is active only after myocyte differentiation. The MCK promoter resulted in high levels of $\mathrm{Cx} 43$ expression in differentiated myotubes but did not cause cell death during differentiation. MCK-Cx43-transfected myoblasts formed viable cardiac grafts and, in some cases, Cx43-expressing myotubes were in close apposition to host cardiomyocytes, possibly allowing electrical coupling. Thus, high levels of $\mathrm{Cx} 43$ during skeletal muscle differentiation cause cell death. When, however, expression of $\mathrm{Cx} 43$ is delayed until after differentiation, using the MCK promoter, myotubes are viable and express gap junction proteins after grafting in the heart. This strategy may permit electrical coupling of skeletal and cardiac muscle for cardiac repair.
\end{abstract}

\section{OVERVIEW SUMMARY}

Cellular cardiomyoplasty using skeletal myoblasts may be beneficial for infarct repair. Differentiated skeletal muscle, however, lacks gap junction expression, thus preventing electrical coupling to host myocardium. We sought to overexpress the gap junction protein connexin $43(\mathrm{Cx} 43)$ in differentiated skeletal myotubes, using retroviral, adenoviral, and plasmid-mediated gene transfer. All strategies resulted in overexpression of $\mathrm{Cx} 43$ in cultured myotubes, but expression of $\mathrm{Cx} 43$ from constitutive viral promoters caused significant death upon differentiation. Retrovirally transfected myoblasts did not express $\mathrm{Cx} 43$ after grafting into the heart. Adenovirally transfected myoblasts expressed abundant $\mathrm{Cx}_{43}$ in cardiac grafts, but grafts showed signs of cell death. Interestingly, transfection of differentiated myotubes with adenoviral $\mathrm{Cx} 43$ was nontoxic, implying a window of vulnerability during differentiation. Therefore, Cx43 was expressed from the muscle creatine kinase (MCK) promoter, which is active only after myocyte differentiation. The MCK promoter resulted in high levels of $\mathrm{Cx} 43$ expression in differentiated myotubes without causing cell death and MCK-Cx43-transfected myoblasts formed viable cardiac grafts, with structural evidence for gap junctions with host myo-cardium.

\section{INTRODUCTION}

$\mathbf{M}$ YOCARDIAL ISCHEMIA leads to rapid death of cardiomyocytes, and there is no intrinsic repair mechanism in the heart sufficient to restore myocardial function. Instead, scar formation takes place, replacing contractile myocardium with akinetic tissue (Reimer and Jennings, 1979; Soonpaa and 
Field, 1998). Cellular cardiomyoplasty represents a potentially promising strategy to replace the noncontractile fibrous scar tissue with new contractile muscle. Skeletal myoblasts are being explored as a cell source for cardiomyoplasty for several reasons: (1) availability from any given patient, thus providing an autologous, nonimmunogenic source of cells, (2) potent proliferative capacity, (3) resistance to ischemia, and (4) contractility. A major theoretical caveat concerning the use of skeletal muscle cells for cardiac repair is their lack of gap junctions that mediate electrical coupling. In contrast, electrical coupling between heart muscle cells is dependent on connexin43 (Cx43)rich gap junctions (Beyer et al., 1987). Interestingly, skeletal muscle cells express Cx43 as replicating myoblasts but downregulate its expression progressively upon fusion, myotube formation, and further differentiation (Schmalbruch, 1982; Balogh et al., 1993). As a result, mature skeletal myofibers become electrically insulated, permitting fine motor control to be achieved by innervation with motoneurons.

A previous study from our laboratory has shown that primary skeletal myotubes can couple to cardiomyocytes via electromechanical junctions in vitro, thus inducing synchronous, calcium-dependent contractions in the skeletal muscle cells. This indicated that low-level Cx43 expression was sufficient to achieve electrical coupling in vitro. Electromechanical coupling was not detectable in skeletal muscle grafts in the heart, however, because the skeletal muscle cells did not express the critical junctional molecules in vivo (Reinecke et al., 2000). These experiments led us to hypothesize that forced expression of $\mathrm{Cx} 43$ in differentiated skeletal myocytes would allow electrical coupling of these cells to the host cardiomyocytes after cardiac grafting.

\section{MATERIALS AND METHODS}

\section{Strategies to overexpress Cx43 in differentiated skeletal myocytes}

Our initial goal was to generate a myoblast clone with stable expression of the transgene $\mathrm{Cx} 43$ after differentiation. Therefore, we chose the mouse myoblast line MM14 (kindly provided by S. Hauschka, University of Washington, Seattle, WA). The MM14 myoblast line shows growth characteristics similar to those of primary myoblasts but is dependent on basic fibroblast growth factor (bFGF) for continuous proliferation (Linkhart et al., 1981). MM14 myoblasts were used for transduction with a Cx43-retrovirus. To more closely mimic a clinical situation, we also wished to test overexpression of $\mathrm{Cx} 43$ in primary myoblasts. To achieve high-efficiency infection of primary myoblasts we generated a $\mathrm{Cx} 43$-adenovirus and used Fischer neonatal rat primary myoblasts as target cells. The mouse myoblast line $\mathrm{C} 2 \mathrm{C} 12$ (American Type Culture Collection [ATCC], Manassas, VA) was used for plasmid transfections. The culture conditions for the three cell types were as described previously in detail (Reinecke and Murry, 2000). The rat Cx43 cDNA was kindly provided by E.C. Beyer (University of Chicago, Chicago, IL).

\section{Animal procedures}

The study protocol was approved by the Institutional Animal Care and Use Committee of the University of Washington and was conducted in accordance with federal guidelines. Nor- mal hearts of adult male nude mice were injected with a standard dose of $1 \times 10^{6}$ myoblasts suspended in $7 \mu \mathrm{l}$ of medium without serum and antibiotics as previously described in detail (Reinecke and Murry, 2003). Mice were killed 7, 10, or 14 days after grafting and their hearts were fixed in methyl Carnoy's solution and routinely processed for histology as described previously (Murry et al., 1996b; Reinecke and Murry, 2000). Subcutaneous grafting (referred to as "skin grafts") was used as a relatively simple test model to evaluate transgene expression in vivo. Typically, $5 \times 10^{6}$ myoblasts were suspended in $50 \mu \mathrm{l}$ of serum and antibiotic-free medium and injected subcutaneously (4-6 grafts on the back of the mouse). The skin grafts were recovered 1-2 weeks after transplantation and routinely processed for histology (paraffin-embedded or cryosections).

\section{Antibodies and immunocytochemistry}

Immunostaining of deparaffinized sections was performed by routine staining techniques as previously described (Murry et al., 1996b; Reinecke et al., 1999, 2002). A monoclonal connexin43 antibody (Chemicon International, Temecula, CA) was used for single immunostaining (diluted 1:200) and Western blotting (diluted 1:1000). In double-staining reactions sections were first incubated with the MY32 antibody (Havenith et al., 1990) (alkaline phosphatase-conjugated anti-fast skeletal myosin heavy chain, diluted 1:400; Sigma, St. Louis, MO) and then with a polyclonal connexin43 antibody (diluted 1:5000; Chemicon International). Vector red (Vector Laboratories, Burlingame, CA) was used as the first chromogen and diaminobenzidine (DAB; Sigma) was used as the second chromogen.

\section{Cx43 retrovirus production and transduction}

Production of the retrovirus encoding rat $\mathrm{Cx} 43$ closely followed a protocol developed by Miller and colleagues (1993). Briefly, rat Cx43 cDNA was digested with EcoRI and ligated into the EcoRI site of the LXSN retroviral backbone (LTR driving expression of the transgene, $X=$ transgene, SV40 promoter driving the resistance gene, Neomycin phosphotransferase; generously provided by A.D. Miller, Fred Hutchinson Cancer Research Center, Seattle, WA). The resulting LCx43SN plasmid was used for transient transfection of ecotropic PE501 packaging cells. A 24-hr supernatant from these cells was used to infect amphotropic PA317 packaging cells. After 14 days of culture in selection medium containing G418 (Geneticin, 1.6 $\mathrm{mg} / \mathrm{ml}$; Invitrogen, Carlsbad, CA), 12 neomycin-resistant clones were selected for further analysis. Integration of the retroviral construct into genomic DNA was tested by polymerase chain reaction (PCR). For mass production of the $\mathrm{Cx} 43$ retrovirus a positive cell clone was expanded, and the supernatant was collected and used to transduce the mouse myoblast line MM14. For the transduction of MM14 myoblasts the retrovirus-containing supernatant was mixed with basic fibroblast growth factor (bFGF)-containing growth medium (1:1) and MM14 cultures were incubated for $24 \mathrm{hr}$ with the retrovirus. Regular growth medium was then added back. Two days after transduction, selection for neomycin resistance was started by adding G418 (1.6 mg/ml) to the growth medium. Stably transduced MM14 clones were picked after 14 days, using cloning rings. The clones were expanded and analyzed for expression of the $\mathrm{Cx} 43$ transgene after differentiation by Western blot anal- 
ysis and immunostaining. As a control, an empty LXSN retrovirus without transgene was generated and used to derive an "empty" transduced MM14 clone.

\section{Cx43 adenovirus production and infection}

An adenovirus encoding rat $\mathrm{Cx} 43$ driven by the cytomegalovirus (CMV) promoter was generated by using the
AdEasy system originally described by $\mathrm{He}$ and colleagues (1998) (the authors also made detailed protocols available online at http://www.coloncancer.org/adeasy.htm). Rat Cx 43 cDNA was digested, using the $S a l I$ and $X b a I$ sites. The $\mathrm{Cx} 43$ insert was agarose gel purified and ligated into the SalI- and XbaI-digested AdTrack-CMV vector ("Track" refers to green fluorescent protein $[\mathrm{GFP}]$ in the vector driven by its own CMV promoter). The resulting AdTrack-CMV-Cx43 construct was linearized by di-

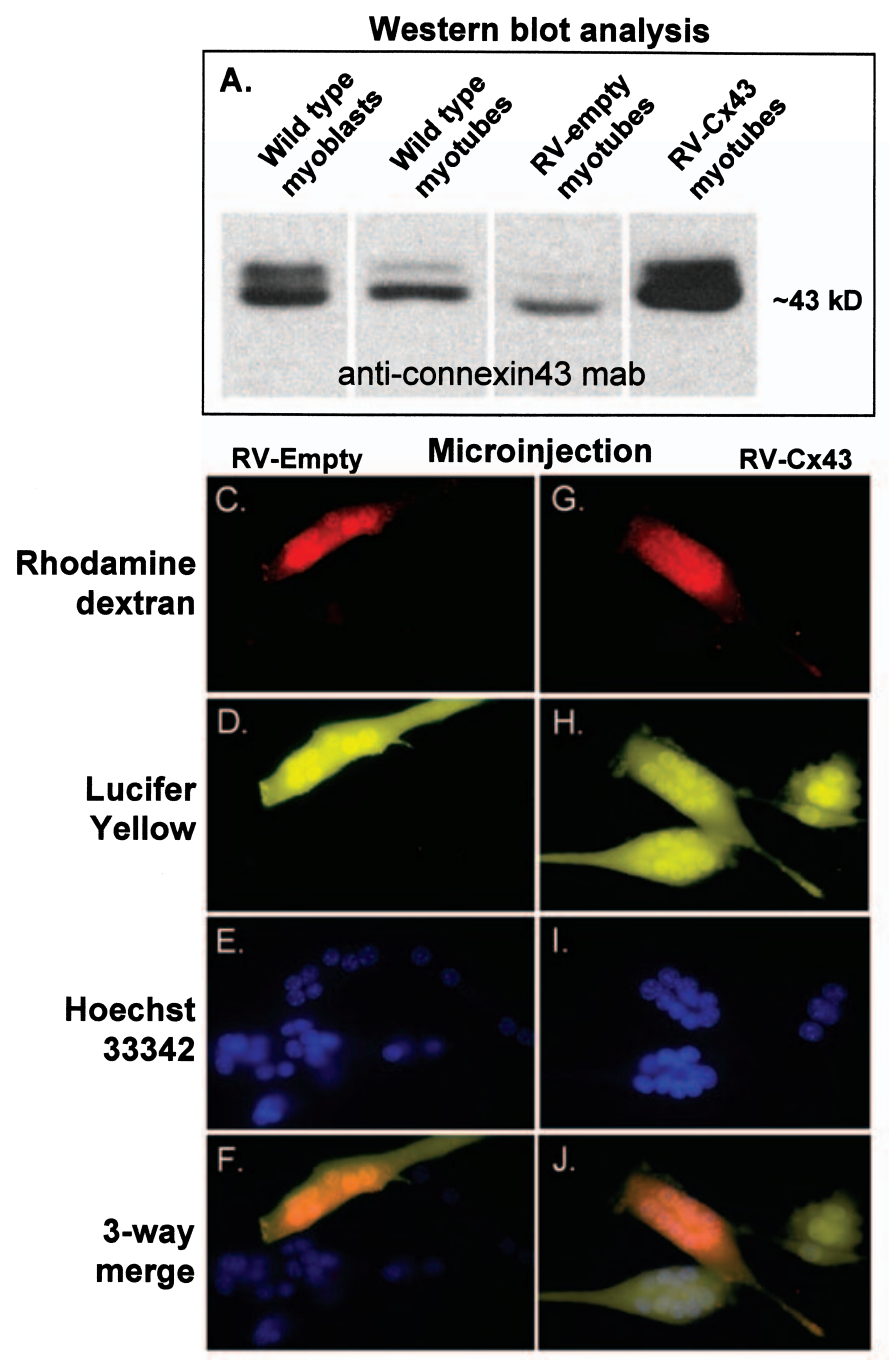

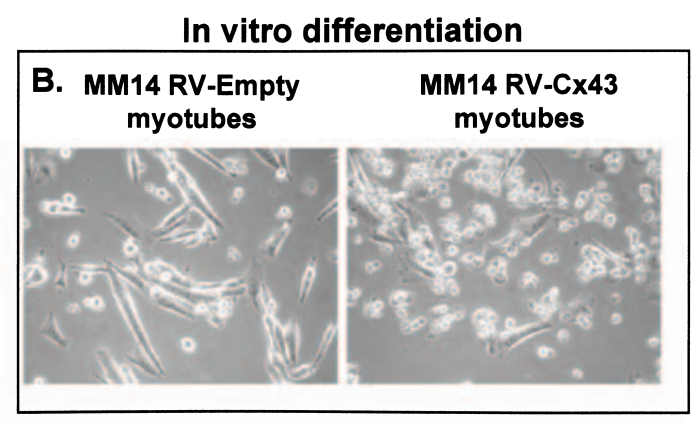

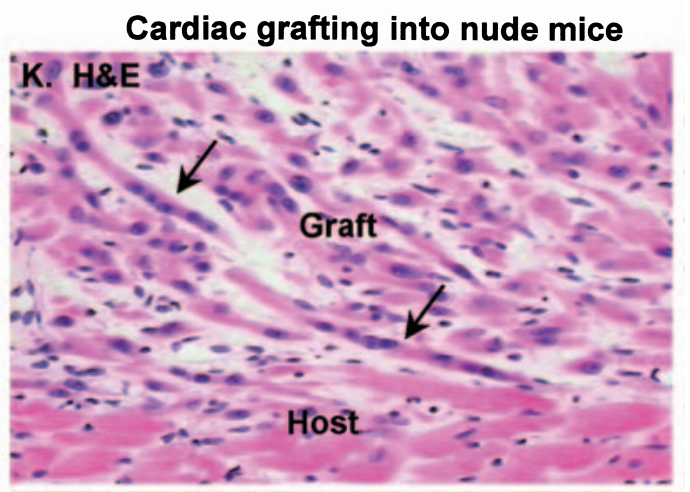

L. Connexin43

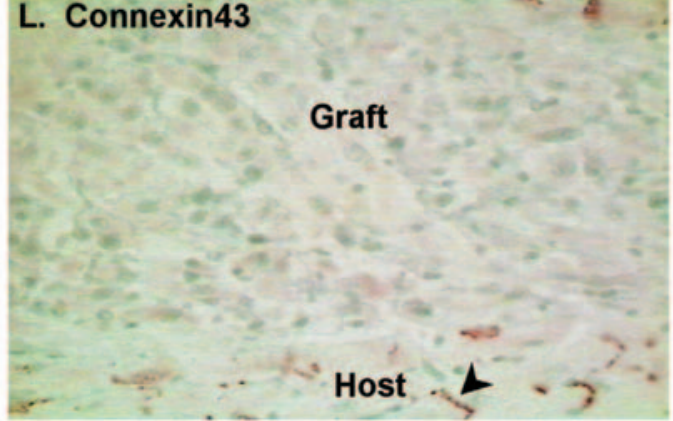

FIG. 1. (A) Western blot analysis of wild-type MM14 myoblasts and myotubes and of MM14 myotubes transduced with an empty retrovirus (control) or a Cx43-retrovirus. MM14 cells express abundant levels of Cx43 and downregulate this gap junction protein as they undergo differentiation. MM14 myotubes transduced with an empty retrovirus (control) show similar downregulation. In contrast, MM14 myotubes previously transduced with a Cx43-retrovirus maintain abundant levels of Cx43 expression. Cell lysates of myotubes were prepared on day 7 after induction of differentiation. (B) MM14 cultures on day 3 after induction of differentiation. MM14 myoblasts transduced with the empty control retrovirus showed normal differentiation with occasional dead (refractile) cells (left). In contrast, in differentiating cultures of Cx43-retrovirus-transduced MM14 cells, increased cell death was observed. The surviving cells appeared as a mixed population of multi- and mononucleated MM14 cells. (C-J) Analysis of gap junction function by microinjection. Myotubes transduced with the empty retrovirus or Cx43-retrovirus were injected with rhodamine dextran (gap junction-impermeable dye) and Lucifer yellow (gap junction-permeable dye) 6 days after induction of differentiation. Dye transfer of Lucifer yellow was absent between myotubes transduced with the empty retrovirus (C-F). In contrast, Cx43-transduced myotubes readily permitted Lucifer yellow dye transfer to adjacent myotubes, suggesting functional gap junctions $(\mathbf{G}-\mathbf{J})$. (K and $\mathbf{L}$ ) Cardiac grafting of MM14 myoblasts transduced with the Cx43-retrovirus into normal nude mouse hearts. On day 10 after grafting, hematoxylin and eosin (H\&E) staining revealed the presence of multinucleated skeletal myotubes (K, arrows). Immunostaining for $\mathrm{Cx} 43$ on consecutive sections, however, failed to detect expression of the transgene in the graft. In contrast, $\mathrm{Cx} 43$-positive gap junctions were readily detected in the intercalated disks of host myocardium (L, arrowhead). Original magnification: $\times 400$. 
gestion with PmeI and subsequently cotransformed, using electroporation-competent Escherichia coli BJ5183 cells with the adenoviral backbone plasmid pAdEasy-1. Recombinants were selected for kanamycin resistance, and recombination was confirmed by restriction endonuclease analyses. Finally, the PacI-linearized recombinant plasmid was transfected into adenovirus packaging cell line 293. Mass production of adenoviruses was performed as described previously (Murry et al., 1996a). The concentration of the adenovirus preparations was measured by spectrophotometry. An optical density (OD) at 260 $\mathrm{nm}$ of 1 equals approximately $10^{12}$ viral particles $/ \mathrm{ml}$. Primary neonatal Fischer rat myoblasts were grown in gelatin-coated 100 -mm plates in F10C supplemented with $15 \%$ horse serum, bFGF (6 ng/ml), and antibiotics (Murry et al., 1996b; Reinecke and Murry, 2000). The day before grafting, the myoblast cultures were infected with 500 viral particles/cell in $5 \mathrm{ml}$ of medium for $2.5 \mathrm{hr}$ with agitation in a humidified incubator $\left(37^{\circ} \mathrm{C}, 5 \% \mathrm{CO}_{2}\right)$. Cell viability was confirmed the next day by trypan blue staining, and myoblasts were prepared for cardiac injection as described (Murry et al., 1996b; Reinecke and Murry, 2000).

\section{Muscle creatine kinase promoter-Cx43 plasmid vector}

The backbone of this construct was kindly provided by $\mathrm{S}$. Hauschka (University of Washington, Seattle, WA) and consisted of a full-length muscle creatine kinase (MCK) promoter (Jaynes et al., 1986, 1988), a multicloning site, and kanamycin and neomycin resistance genes for selection in prokaryotes and eukaryotes, respectively. The MCK gene is inactive in myoblasts but is transcriptionally activated during striated muscle differentiation and is expressed at high levels in adult heart and skeletal muscles (Chamberlain et al., 1985; Lyons et al., 1991). Thus, on differentiation the Cx43 transgene becomes activated whereas the endogenous $\mathrm{Cx} 43$ gene expression is muted. This vector was digested, utilizing the NotI and AgeI sites. Compatible NotI and AgeI sites for Cx43 were generated by PCR. The PCR product was agarose gel purified, and a mutation-free clone was obtained after sequencing, termed pMCK-Cx43. C2C12 myoblasts were transfected with pMCK-Cx43, using
SuperFect transfection reagent (Qiagen, Valencia, CA). Stable lines were selected on the basis of neomycin resistance (G418, $1.6 \mathrm{mg} / \mathrm{ml})$.

\section{Cx43 expression screening}

To screen for the expression and function of the Cx43 transgene in vitro we employed Western blot analysis, immunostaining, and microinjection (dye transfer) as described (Reinecke and Murry, 2000; Reinecke et al., 2000). For Western blot analysis typically $80-100 \mu \mathrm{g}$ of protein from a total cell lysate was subjected to sodium dodecyl sulfate-polyacrylamide gel electrophoresis (SDS-PAGE) and blotted, and the membrane was probed with a monoclonal anti-Cx43 antibody. For immunocytochemistry cultures were routinely fixed with icecold $100 \%$ methanol. If detection of both GFP and connexin43 immunostaining was required, cultures were fixed for $2 \mathrm{~min}$ in $3 \%$ paraformaldehyde, $0.2 \%$ Triton $\mathrm{X}-100$, and $5 \mathrm{~m} M$ EGTA in phosphate-buffered saline (PBS) ( $\mathrm{pH} 7.2$ ), rinsed with PBS, and then fixed for $20 \mathrm{~min}$ in 3\% paraformaldehyde. Connexin43 was detected with a mouse monoclonal antibody (diluted 1:200; Chemicon International). Microinjection studies were performed to evaluate functional gap junctions between differentiated myotubes as described previously (Reinecke et al., 2000). The injected dye solution was composed of $10 \%$ Lucifer yellow (gap junction permeable) and $10 \%$ tetramethylrhodamine-dextran (gap junction impermeable) (Molecular Probes, Eugene, OR). The injected cultures were fixed with $4 \%$ paraformaldehyde and cell nuclei were counterstained with Hoechst 33342 (Molecular Probes).

\section{RESULTS}

\section{Retrovirus-mediated Cx43 expression in MM14 myoblasts}

We established several lines of MM14 mouse myoblasts transduced with the $\mathrm{Cx} 43$ retrovirus. Expression of the transgene was driven by the viral long terminal repeat (LTR) promoter. It is known that primary myoblasts as well as immor-

FIG. 2. In vitro characterization of primary Fischer rat myoblasts infected with GFP-adenovirus (control) or Cx43-GFP-adenovirus and then differentiated for 5 days. Ad-GFP-infected myotubes did not express Cx43 (A-D). In contrast, Cx43 expression was readily detected in differentiated myotubes infected with the Cx43-adenovirus (E-H). Both control (I-L) and Cx43-adenovirus (M-P)-infected myotubes expressed fast skeletal muscle-specific myosin heavy chain (MHC fast; detected with the MY32 antibody; Sigma) (Havenith et al., 1990). Green, GFP fluorescence; blue, Hoechst 33342 used as a nuclear counterstain. Original magnification: $\times 400$.

FIG. 3. Primary Fischer rat myoblasts were infected with GFP-adenovirus (control) or Cx43-GFP adenovirus and grafted subcutaneously into nude mice (skin graft model). Myoblasts infected with control GFP-adenovirus gave rise to differentiated skeletal muscle grafts (A), whereas myoblasts infected with the Cx43-adenovirus (also encoding GFP) did not yield viable grafts (B). Green, GFP fluorescence; blue, Hoechst 33342 used as a nuclear counterstain. (C-F) Interestingly, if already differentiated myotubes were infected in vitro, both GFP-adenovirus-infected myotubes (C and $\mathbf{D})$ and $\mathbf{C x} 43$-adenovirus-infected myotubes (E and F) appeared viable. Top: Phase-contrast. Bottom: GFP fluorescence (green). Primary Fischer rat myoblasts infected with lowdose $\mathrm{Cx} 43$-adenovirus were grafted into normal nude mouse hearts. (G) At 1 week after grafting hematoxylin and eosin staining revealed the presence of multinucleated skeletal myotubes $(\mathbf{G}$, inset $)$. Immunostaining for Cx43 on consecutive sections detected abundant expression of the transgene in the graft (brown) (H). Possible sites of gap junctional communication between graft myotubes and host cardiomyocytes were rarely observed (I and J, arrowheads). Many Cx43-expressing myotubes, however, showed signs of cell injury, including swelling of subcellular compartments $(\mathbf{K}$ and $\mathbf{L})$. Original magnification: $(\mathbf{A}$ and $\mathbf{B}) \times 400$; $(\mathbf{C}-\mathbf{F}) \times 200 ;(\mathbf{G}$ and $\mathbf{H}) \times 40 ;(\mathbf{G}$, inset, and $\mathbf{I}-\mathbf{L}) \times 1000$. 


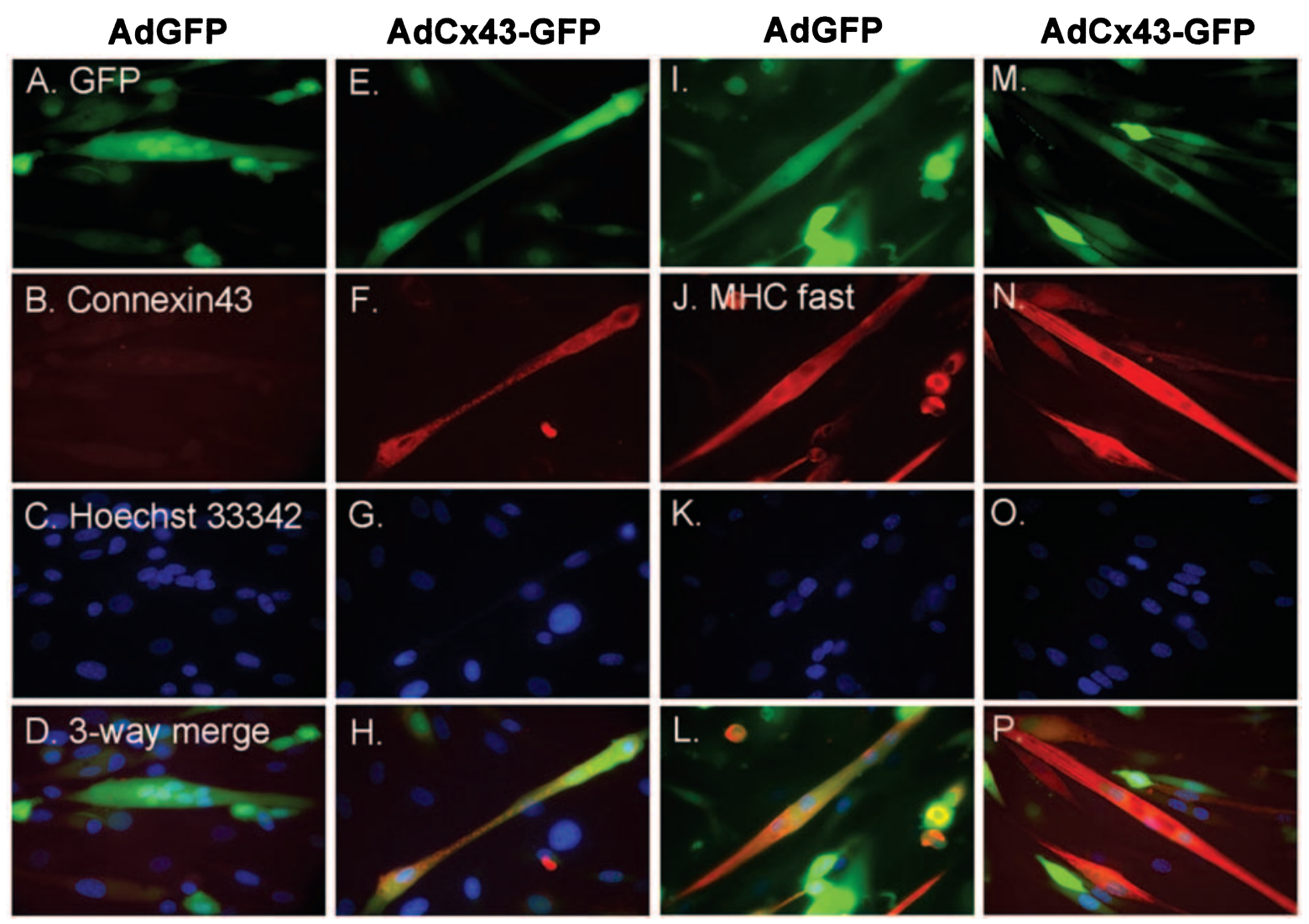

FIG. 2.
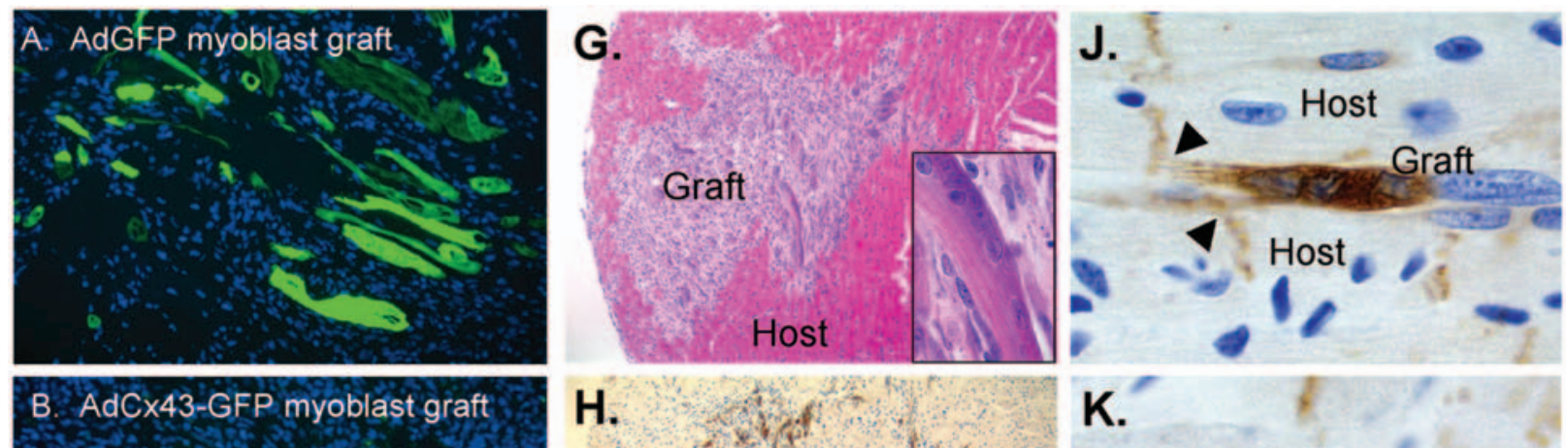

K.

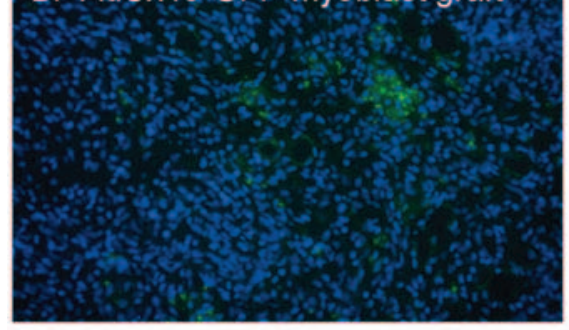

H.
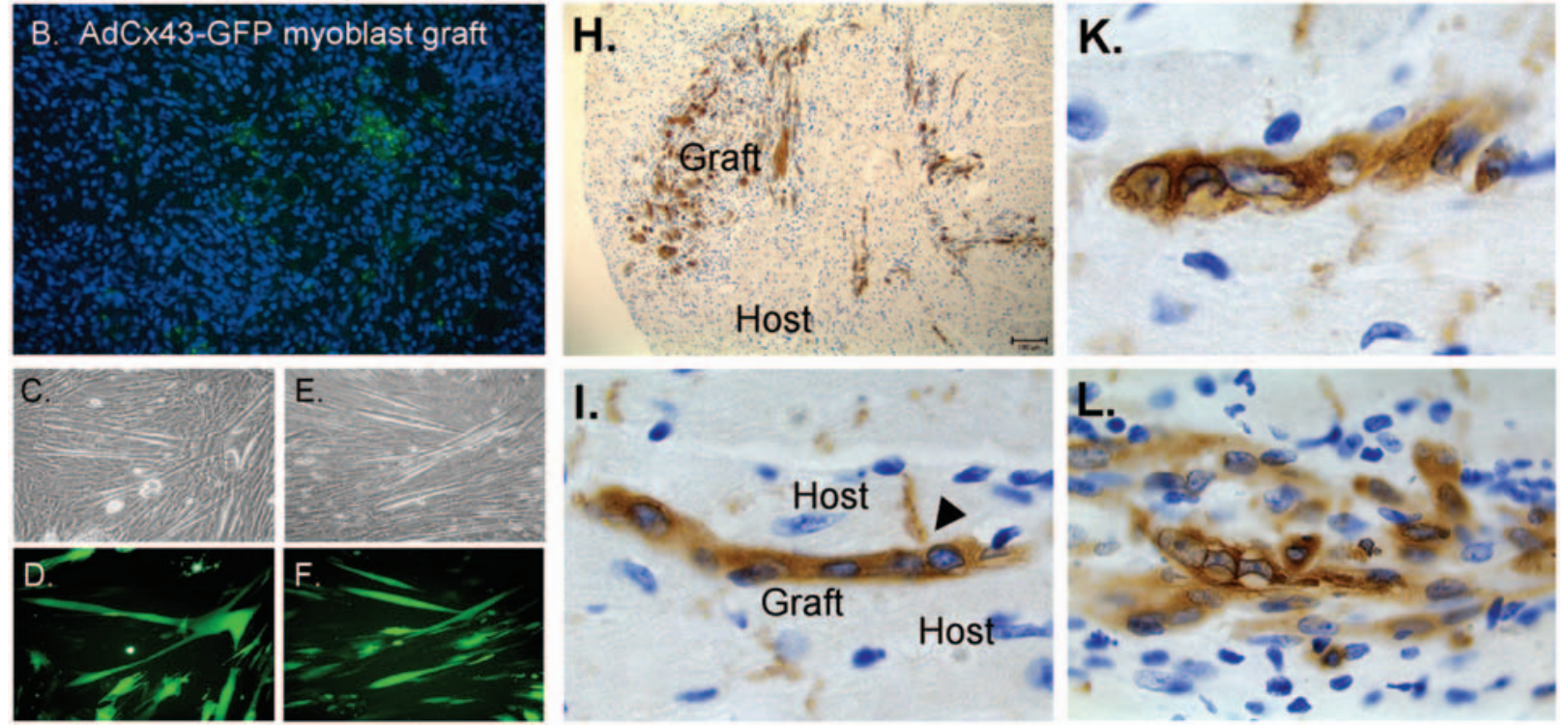

FIG. 3. 
talized myoblast lines express endogenous $\mathrm{Cx} 43$, which appears to be required for normal fusion (Mege et al., 1994). Therefore, we analyzed the expression levels of transgenic Cx43 in differentiated myotubes by Western blot analysis (Fig. 1A). Nontransfected (wild-type) myoblasts expressed abundant levels of $\mathrm{Cx} 43$, and this was significantly downregulated upon differentiation into myotubes. Myotubes that had been previously transduced with a control (empty) retrovirus showed similarly low levels of $\mathrm{Cx} 43$ protein. In contrast, myoblasts transduced with the Cx43-encoding retrovirus showed high levels of Cx43 expression after differentiating into myotubes.

In myoblasts transduced with the $\mathrm{Cx} 43$-retrovirus we noticed increased cell death upon differentiation (Fig. 1B). In contrast, a myoblast clone transduced with the control (empty) retrovirus showed normal fusion and differentiation, thus excluding retroviral transduction itself as the cause of increased cell death. Microinjection studies performed on surviving myotubes showed that $\mathrm{Cx} 43$-transduced myotubes permitted Lucifer yellow dye transfer (Fig. 1G-J). In contrast, dye transfer was not observed in myotubes transduced with an empty retrovirus (Fig. 1C-F). In both cases, however, microinjection of MM14 myotubes frequently resulted in hypercontraction and subsequent detachment from the surface. A clone showing robust $\mathrm{Cx} 43$ expression levels in differentiated myotubes was chosen for grafting studies. Both MM14 myoblasts transduced with empty retrovirus (control) and MM14 myoblasts transduced with $\mathrm{Cx} 43$ retrovirus gave rise to viable grafts in nude mouse hearts. We were not able, however, to detect $\mathrm{Cx} 43$ expression in the MM14-Cx43 grafts 10 days $(n=7)$ (Fig. $1 \mathrm{~K}$ and L) and 4 weeks $(n=4)$ after grafting. The absence of $\mathrm{Cx} 43$ expression suggests possible silencing of the retroviral LTR promoter in vivo. Extinction of retroviral vector expression has been previously observed after implantation of transduced embryonic and hematopoietic stem cells as well as fibroblasts, myoblasts, and hepatocytes (Lund et al., 1996; Cherry et al., 2000).

\section{Adenovirus-mediated Cx43 expression in primary Fischer rat myoblasts}

The rationale behind using primary myoblasts as target cell for connexin43 gene transfer is given by the clinical situation. Here, one would need to isolate satellite cells from a given patient and genetically modify these cells efficiently. To mimic this situation, we generated an adenovirus encoding $\mathrm{Cx} 43$ (and also GFP, each driven by separate CMV promoters) and infected cultures of undifferentiated primary Fischer rat myoblasts. Immunostaining demonstrated the expression of connexin43 in differentiated myotubes infected with the $\mathrm{Cx} 43$-adenovirus (Fig. $2 \mathrm{E}-\mathrm{H})$ but not in myotubes infected with the control GFP-adenovirus (Fig. 2A-D). Both the GFP- and Cx43-GFP-infected myotubes expressed typical muscle markers such as desmin (data not shown) and fast skeletal myosin heavy chain (Fig. 2I-P).

Similar to our observations with the Cx43-retrovirus, we noticed increased cell death on differentiation in cultures infected with $\mathrm{Cx} 43$-adenovirus but not in cultures infected with a GFPadenovirus (data not shown). To test whether Cx43-expressing myoblasts showed similar susceptibility to death in vivo, primary myoblast cultures were infected with the control GFP-adenovirus or the Cx43-adenovirus and then grafted subcutaneously into nude mice. Figure $3 \mathrm{~A}$ shows that myoblasts infected with the control GFP-adenovirus gave rise to green fluorescent differentiated myofibers. In contrast, myoblasts infected with the $\mathrm{Cx} 43$-adenovirus did not yield viable grafts (Fig. 3B). Interestingly, if already differentiated cultures were infected with the $\mathrm{Cx} 43$ adenovirus, no increase in cell death was observed in vitro, and infected myotubes appeared healthy (Fig. 3C-F).

Reduction in cell death by transfection after differentiation suggests that $\mathrm{Cx} 43$ levels need to be tightly regulated during critical steps in the differentiation process or otherwise may have adverse effects on cell survival. Unfortunately, trypsinizing differentiated primary myotubes (as necessary for grafting experiments) causes massive cell death. Therefore, we infected undifferentiated myoblasts with the Cx43-adenovirus at $\sim 30 \%$ efficiency, reasoning that this relatively low infection rate would yield lower, nontoxic Cx43 levels, yet result in a high percentage of $\mathrm{Cx} 43$-positive myotubes due to fusion of infected with noninfected myoblasts. One week after grafting into normal nude mouse hearts the Cx43-infected myoblasts had differentiated to form myotubes (Fig. 3G and inset). Despite the relatively low infection rate, staining for $\mathrm{Cx} 43$ revealed abundant expression of the transgene (Fig. $3 \mathrm{H}$ ). Although $\mathrm{Cx} 43$ is typically localized to the cell membrane, the transfected myotubes demonstrated diffuse cytoplasmic staining. In rare cases, we observed possible sites of gap junctional communication between host and graft (Fig. 3I and J). Many of the Cx43-expressing graft myotubes appeared atrophic and had perinuclear vacuoles (Fig. 3K and L), suggesting problems with intracellular volume regulation.

\section{Expression of muscle creatine kinase promoter-CX43 in C2C12 myoblasts}

Previous studies indicated that overexpressing Cx43 during muscle differentiation caused cell death, but that $\mathrm{AdCx} 43$ infection of already differentiated myotubes was well tolerated. We therefore hypothesized that a promoter active only in differentiated skeletal muscle would result in myotubes that expressed $\mathrm{Cx} 43$ in a nontoxic fashion. We therefore selected the muscle creatine kinase (MCK) promoter, which is transcriptionally activated during striated muscle differentiation and is expressed at high levels in adult heart and skeletal muscles (Chamberlain et al., 1985; Lyons et al., 1991). Thus, the Cx43 transgene should be active only in differentiated myocytes (while the endogenous $\mathrm{Cx} 43$ gene expression is muted), but not in undifferentiated myoblasts. It is also known that the fulllength 3.3-kb MCK promoter, as used in our construct, is approximately $8 \%$ as active as the CMV promoter in myocytes (Hauser et al., 2000).

C2C12 myoblasts stably transfected with the MCK-Cx43 plasmid showed normal growth, and no increase in cell death was observed on differentiation (Fig. 4A-C). Western blot analysis of cell lysates prepared from day 7 differentiated wild-type and Cx43-transfected myotubes revealed persistent overexpression of the transgene (Fig. 4D). Several MCK-Cx43-expressing $\mathrm{C} 2 \mathrm{C} 12$ clones were then tested in the subcutaneous graft model and grafts were analyzed at 1 week. In most clones expression of the Cx43 transgene was readily detectable, indicating that this mammalian promoter was not silenced in vivo (data not shown). The clone with the most abundant expression 
was then grafted into normal nude mouse hearts. In contrast to myoblasts infected with the Cx43 adenovirus, the MCK-Cx43transfected myoblasts formed viable, differentiated grafts even 2 weeks after grafting. The grafts robustly expressed Cx43, and in some cases there was evidence of $\mathrm{Cx} 43$-positive junction formation between graft myotubes and host cardiomyocytes (Fig.
$4 \mathrm{E}$ and F). These experiments suggest that expression of $\mathrm{Cx} 43$ in differentiated myotubes may be consistent with survival and functional integrity of skeletal myocytes.

Finally, we tested whether differences in the susceptibility to death could be attributed to different promoters (LTR, CMV versus MCK) and timing of Cx43 transgene expression or sim-
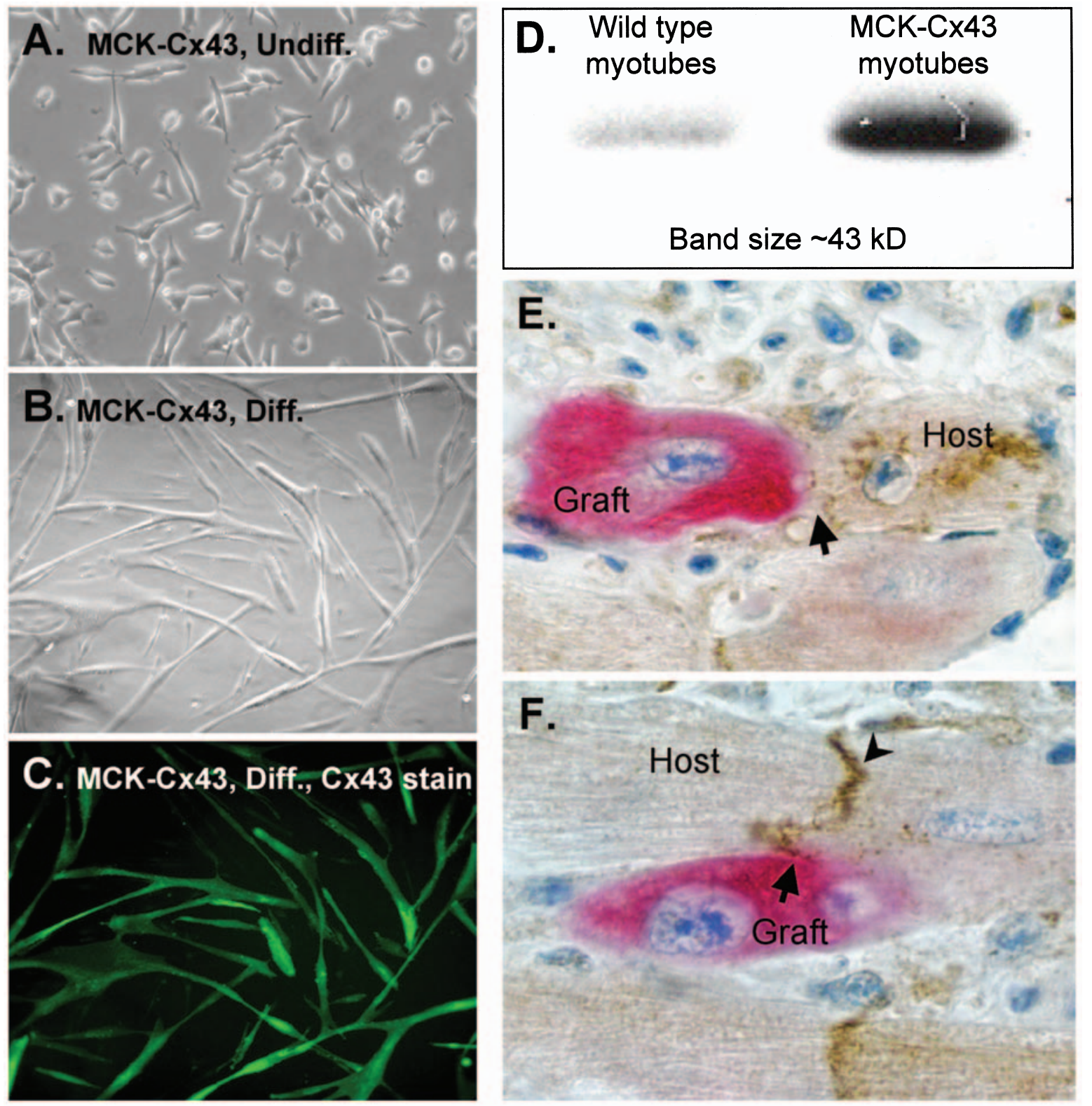

FIG. 4. Characterization of an MCK-Cx43-overexpressing $\mathrm{C} 2 \mathrm{C} 12$ clone. Phase-contrast photomicrographs of stably transfected undifferentiated myoblasts (A) and subsequently differentiated, day 7 myotubes (B). Immunostaining for Cx43 showed abundant expression of the transgene in the differentiated C2C12 myotubes (C) (mouse monoclonal Cx43 antibody [Chemicon International]; rabbit anti-mouse FITC-conjugated secondary antibody). Western blot analysis (D) of wild-type C2C12 myotubes and C2C12 cells transfected with MCK-Cx43 plasmid. Wild-type C2C12 myotubes expressed low but detectable levels of Cx43. In contrast, transfected cells expressed elevated levels of the $\mathrm{Cx} 43$ transgene. Cell lysates of myotubes were prepared on day 7 after induction of differentiation. Cardiac grafting of $\mathrm{C} 2 \mathrm{C} 12$ myoblasts transfected with the MCK-Cx43 plasmid into normal nude mouse hearts ( $\mathbf{E}$ and $\mathbf{F}$ ). Heart sections were immunostained for fast skeletal myosin heavy chain (red) and Cx43 (brown) 1 week after grafting. In some cases $\mathrm{Cx} 43$ was localized at the interface between graft myotubes (graft) and host cardiomyocytes (host), suggesting gap junctional coupling ( $\mathbf{E}$ and $\mathbf{F}$, arrows). The arrowhead in (F) indicates the connexin43-positive intercalated disk between two coupled host cardiomyocytes. Original magnification: $(\mathbf{A}-\mathbf{C}) \times 200 ;(\mathbf{E}$ and $\mathbf{F}) \times 1000$. 
ply the different cell types used. We therefore infected wildtype C2C12 myoblasts with AdGFP or AdCx43-GFP and switched the cultures to differentiation $24 \mathrm{hr}$ after infection. Similar to our previous results with primary myoblasts, AdCx43-infected $\mathrm{C} 2 \mathrm{C} 12$ cells showed increased cell death compared with AdGFP-infected cultures (data not shown). In contrast, if differentiated $\mathrm{C} 2 \mathrm{C} 12$ myotubes were infected with the $\mathrm{Cx} 43$ adenovirus no increase in cell death was observed, again suggesting that the right timing of $\mathrm{Cx} 43$ overexpression is critical in skeletal muscle cells.

\section{DISCUSSION}

In the present study we investigated overexpression of the gap junction molecule connexin43 in skeletal muscle cells, the rationale being the use of these cells for cardiac repair (cellular cardiomyoplasty). In contrast to skeletal muscle, the proper function of cardiac muscle heavily relies on the presence of gap junctions. Before fusion, myoblasts predominantly express the endogenous $\mathrm{Cx} 43$ gap junction protein, but this expression is downregulated once fusion is initiated (Balogh et al., 1993). Therefore, our goal was to achieve overexpression of connexin43 in differentiated skeletal myotubes. The major findings are as follows: (1) constitutive expression of $\mathrm{Cx} 43$ in myoblasts appears to interfere with cell fusion and survival; and (2) overexpression of connexin43 in differentiated myotubes may be achieved by using promoters active only in differentiated skeletal myocytes (such as the muscle creatine kinase promoter) or transfection of already differentiated myotubes.

Using three different strategies, that is, retrovirus-, adenovirus-, and plasmid-based vectors encoding rat connexin43 (Beyer et $a l .$, 1987), we achieved overexpression in undifferentiated myoblasts (mouse $\mathrm{C} 2 \mathrm{C} 12$ and MM14 cells, and neonatal rat primary myoblasts) without apparent negative effects on the transfected cells under growth conditions. Expression of the Cx43 transgene was also tolerated when already differentiated myotubes were transfected or when connexin 43 expression was driven by the muscle creatine kinase promoter. It is important to note that the MCK promoter is inactive in myoblasts but is transcriptionally activated during striated muscle differentiation (Chamberlain et al., 1985; Lyons et al., 1991). Thus, both conditions yielded expression of the $\mathrm{Cx} 43$ transgene in differentiated myotubes.

In contrast, massive cell death during differentiation was observed when undifferentiated myoblasts were transfected with constitutively active connexin43 vectors and then subjected to differentiation. Surviving myotubes, however, permitted dye transfer of Lucifer yellow (Fig. 1), indicating functional integrity of the rat $\mathrm{Cx} 43$ transgene in mouse cells. In vivo after cardiac grafting, we failed to detect any connexin43 expression in MM14-derived grafts, possibly due to silencing of the retroviral LTR promoter in vivo as reported in other systems (Lund et al., 1996; Cherry et al., 2000) or selective survival of myotubes with low expression levels, thus eluding immunodetection. The latter appears less likely, however, given the robust Cx43 protein levels (by Western blot) detected in surviving Cx43-transduced myotubes. Similarly, primary myoblasts and C2C12 myoblasts infected with the CMV-Cx43 adenovirus showed increased cell death upon differentiation in vitro and in vivo after subcutaneous or cardiac grafting. Taken together, these results suggest that constitutively high levels of Cx43 may interfere with critical steps during myoblast fusion.

Gap junctions are found in nearly all mammalian tissues, with adult skeletal muscle being one notable exception, although they are present in early developing myoblasts (Schmalbruch, 1982; Balogh et al., 1993). The differentiation of cultured skeletal myoblasts is commonly activated by growth factor withdrawal and accompanied by transcriptional activation of muscle-specific genes, growth arrest, and fusion to form multinucleated myotubes (Olson, 1992, 1993). The muscle-specific basic helix-loop-helix transcription factors, including MyoD, Myf5, myogenin, and MRF4, orchestrate the entire expression program of the various muscle-specific genes, fast skeletal myosin heavy chain and muscle creatine kinase. In our study we successfully used the muscle creatine kinase promoter to drive expression of $\mathrm{Cx} 43$. The MCK-Cx43-transfected cells proliferated and differentiated normally and the $\mathrm{Cx} 43$ transgene was expressed in myotubes in vitro and in vivo after subcutaneous or cardiac grafting. Because the MCK promoter is only approximately $8 \%$ as active as the CMV promoter in myocytes (Hauser et al., 2000), one might speculate that differences in the transcriptional activity of the MCK versus viral promoters (LTR, CMV) may have caused the different outcomes. At least for CMV-driven Cx43 this appears unlikely, because differentiated myotubes transfected with CMV-Cx43 adenovirus appeared viable. It then becomes likely that the timing of $\mathrm{Cx} 43$ transgene expression plays the more important role. Our results suggest that improper levels of $\mathrm{Cx} 43$ at the time of transition from proliferation to differentiation triggered cell death. This could explain the successful expression of the Cx43 transgene in undifferentiated myoblasts maintained under conditions promoting proliferation and in differentiated myotubes (by transfection of already differentiated myotubes or by using the MCK promoter).

Our data contrast somewhat with a previous study by Suzuki and colleagues, who also sought to enhance intercellular communication of skeletal with cardiac myocytes (Suzuki et al., 2001). These authors overexpressed Cx43 in L6 rat skeletal myoblasts, using the human CMV promoter, the same promoter used in our adenovirus approach. They reported that Cx43transfected myoblasts more rapidly fused and differentiated into multinucleated myotubes. It is possible that the L6 cell line used in the Suzuki et al. study may be less susceptible to the detrimental effects of $\mathrm{Cx} 43$ overexpression that we observed. On the other hand, most of their presented data documented successful overexpression of the transgene in undifferentiated myoblasts. In agreement with the current work, overexpression of Cx43 by myoblasts was well tolerated.

Proper function of cardiac muscle relies on the presence of gap junctions located in the intercalated disks of neighboring cardiomyocytes. Despite the fact that mature skeletal myocytes do not express gap junction molecules, they have become a major focus of cellular cardiomyoplasty (Leobon et al., 2003). Currently, autologous skeletal myoblast transplantation for cardiac infarct repair is undergoing clinical trials (Menasche et al., 2001, 2003; Pagani et al., 2003). We hypothesized that expression of gap junctions would enable skeletal muscle cells to electrically couple to host cardiomyocytes, thus providing a physiologically more potent graft cell for the heart. Although 
retrovirally transduced skeletal muscle cells did not express Cx43 after cardiac grafting (Fig. 1), primary myoblasts infected with the $\mathrm{Cx} 43$-adenovirus gave rise to intensely $\mathrm{Cx} 43$-positive cardiac grafts (Fig. 3). Furthermore, we observed Cx43-positive myotubes in close apposition to host cardiomyocytes, perhaps allowing for coupling. Many graft cells expressing AdCMV-Cx43, however, showed signs of cell injury, including swelling of subcellular compartments (Fig. 3). The most promising results were obtained with the muscle creatine kinase promoter driving expression of $\mathrm{Cx} 43$ in differentiated skeletal muscle cells. When these cells were grafted we observed myotubes in close apposition with host cardiomyocytes, and in some cases $\mathrm{Cx} 43$ immunostaining was suggestive of coupling between host and graft via Cx43-positive junctions (Fig. 4). Physiological studies will be required to test definitively whether such coupling has occurred (Rubart et al., 2003).

In summary, we have shown that overexpression of the major gap junction protein of the heart, $\mathrm{Cx} 43$, is feasible in skeletal muscle cells. Our experiments suggest that $\mathrm{Cx} 43$ transgene expression is consistent with cell survival in differentiated cells. In contrast, high levels of transgenic $\mathrm{Cx} 43$ in myoblasts undergoing differentiation appeared to be toxic, leading to cell death. We believe that myoblasts expressing Cx43 under the control of the MCK promoter will allow us to investigate whether gap junction formation between cardiac and skeletal muscle can indeed take place, and if so, whether such coupling will promote contractile function or exert arrhythmic potential.

\section{ACKNOWLEDGMENTS}

This work was supported by NIH grants HL61553, HL64387, and HL03174. We thank Veronica Poppa for excellent technical assistance.

\section{REFERENCES}

BALOGH, S., NAUS, C.C., and MERRIFIELD, P.A. (1993). Expression of gap junctions in cultured rat L6 cells during myogenesis. Dev. Biol. 155, 351-360.

BEYER, E.C., PAUL, D.L., and GOODENOUGH, D.A. (1987). Connexin43: A protein from rat heart homologous to a gap junction protein from liver. J. Cell Biol. 105, 2621-2629.

CHAMBERLAIN, J.S., JAYNES, J.B., and HAUSCHKA, S.D. (1985). Regulation of creatine kinase induction in differentiating mouse myoblasts. Mol. Cell. Biol. 5, 484-492.

CHERRY, S.R., BINISZKIEWICZ, D., VAN PARIJS, L., BALTIMORE, D., and JAENISCH, R. (2000). Retroviral expression in embryonic stem cells and hematopoietic stem cells. Mol. Cell. Biol. 20, 7419-7426.

HAUSER, M.A., ROBINSON, A., HARTIGAN-O'CONNOR, D., WILLIAMS-GREGORY, D.A., BUSKIN, J.N., APONE, S., KIRK, C.J., HARDY, S., HAUSCHKA, S.D., and CHAMBERLAIN, J.S. (2000). Analysis of muscle creatine kinase regulatory elements in recombinant adenoviral vectors. Mol. Ther. 2, 16-25.

HAVENITH, M.G., VISSER, R., SCHRIJVERS-VAN SCHENDEL, J.M., and BOSMAN, F.T. (1990). Muscle fiber typing in routinely processed skeletal muscle with monoclonal antibodies. Histochemistry $\mathbf{9 3}, \mathbf{4 9 7 - 4 9 9}$

HE, T.C., ZHOU, S., DA COSTA, L.T., YU, J., KINZLER, K.W., and
VOGELSTEIN, B. (1998). A simplified system for generating recombinant adenoviruses. Proc. Natl. Acad. Sci. U.S.A. 95, 2509-2514.

JAYNES, J.B., CHAMBERLAIN, J.S., BUSKIN, J.N., JOHNSON, J.E., and HAUSCHKA, S.D. (1986). Transcriptional regulation of the muscle creatine kinase gene and regulated expression in transfected mouse myoblasts. Mol. Cell. Biol. 6, 2855-2864.

JAYNES, J.B., JOHNSON, J.E., BUSKIN, J.N., GARTSIDE, C.L., and HAUSCHKA, S.D. (1988). The muscle creatine kinase gene is regulated by multiple upstream elements, including a muscle-specific enhancer. Mol. Cell. Biol. 8, 62-70.

LEOBON, B., GARCIN, I., MENASCHE, P., VILQUIN, J.T., AUDINAT, E., and CHARPAK, S. (2003). Myoblasts transplanted into rat infarcted myocardium are functionally isolated from their host. Proc. Natl. Acad. Sci. U.S.A. 100, 7808-7811.

LINKHART, T.A., CLEGG, C.H., and HAUSCHKA, S.D. (1981) Myogenic differentiation in permanent clonal mouse myoblast cell lines: Regulation by macromolecular growth factors in the culture medium. Dev. Biol. 86, 19-30.

LUND, A.H., DUCH, M., and PEDERSEN, F.S. (1996). Transcriptional silencing of retroviral vectors. J. Biomed. Sci. 3, 365-378.

LYONS, G.E., MUHLEBACH, S., MOSER, A., MASOOD, R., PATERSON, B.M., BUCKINGHAM, M.E., and PERRIARD, J.C. (1991). Developmental regulation of creatine kinase gene expression by myogenic factors in embryonic mouse and chick skeletal muscle. Development 113, 1017-1029.

MEGE, R.M., GOUdOU, D., GIAUME, C., NICOLET, M., and RIEGER, F. (1994). Is intercellular communication via gap junctions required for myoblast fusion? Cell Adhes. Commun. 2, 329-343.

MENASCHE, P., HAGEGE, A.A., SCORSIN, M., POUZET, B., DESNOS, M., DUBOC, D., SCHWARTZ, K., VILQUIN, J.T., and MAROLLEAU, J.P. (2001). Myoblast transplantation for heart failure. Lancet 357, 279-280.

MENASCHE, P., HAGEGE, A.A., VILQUIN, J.T., DESNOS, M., ABERGEL, E., POUZET, B., BEL, A., SARATEANU, S., SCORSIN, M., SCHWARTZ, K., BRUNEVAL, P., BENBUNAN, M., MAROLLEAU, J.P., and DUBOC, D. (2003). Autologous skeletal myoblast transplantation for severe postinfarction left ventricular dysfunction. J. Am. Coll. Cardiol. 41, 1078-1083.

MILLER, A.D., MILLER, D.G., GARCIA, J.V., and LYNCH, C.M. (1993). Use of retroviral vectors for gene transfer and expression. Methods Enzymol. 217, 581-599.

MURRY, C.E., KAY, M.A., BARTOSEK, T., HAUSCHKA, S.D., and SCHWARTZ, S.M. (1996a). Muscle differentiation during repair of myocardial necrosis in rats via gene transfer with MyoD. J. Clin. Invest. 98, 2209-2217.

MURRY, C.E., WISEMAN, R.W., SCHWARTZ, S.M., and HAUSCHKA, S.D. (1996b). Skeletal myoblast transplantation for repair of myocardial necrosis. J. Clin. Invest. 98, 2512-2523.

OLSON, E.N. (1992). Interplay between proliferation and differentiation within the myogenic lineage. Dev. Biol. 154, 261-272.

OLSON, E.N. (1993). Signal transduction pathways that regulate skeletal muscle gene expression. Mol. Endocrinol. 7, 1369-1378.

PAGANI, F.D., DERSIMONIAN, H., ZAWADZKA, A., WETZEL, K., EDGE, A.S., JACOBY, D.B., DINSMORE, J.H., WRIGHT, S., ARETZ, T.H., EISEN, H.J., and AARONSON, K.D. (2003). Autologous skeletal myoblasts transplanted to ischemia-damaged myocardium in humans: Histological analysis of cell survival and differentiation. J. Am. Coll. Cardiol. 41, 879-888.

REIMER, K.A., and JENNINGS, R.B. (1979). The "wavefront phenomenon" of myocardial ischemic cell death. II. Transmural progression of necrosis within the framework of ischemic bed size (myocardium at risk) and collateral flow. Lab. Invest. 40, 633-644.

REINECKE, H., and MURRY, C.E. (2000). Transmural replacement of myocardium after skeletal myoblast grafting into the heart. Too much of a good thing? Cardiovasc. Pathol. 9, 337-344. 
REINECKE, H., and MURRY, C.E. (2003). Cell grafting for cardiac repair. Methods Mol. Biol. 219, 97-112.

REINECKE, H., ZHANG, M., BARTOSEK, T., and MURRY, C.E. (1999). Survival, integration, and differentiation of cardiomyocyte grafts: A study in normal and injured rat hearts. Circulation 100, 193-202.

REINECKE, H., MACDONALD, G.H., HAUSCHKA, S.D., and MURRY, C.E. (2000). Electromechanical coupling between skeletal and cardiac muscle: Implications for infarct repair. J. Cell Biol. 149. 731-740.

REINECKE, H., POPPA, V., and MURRY, C.E.(2002). Skeletal muscle stem cells do not transdifferentiate into cardiomyocytes after cardiac grafting. J. Mol. Cell. Cardiol. 34, 241-249.

RUBART, M., PASUMARTHI, K.B., NAKAJIMA, H., SOONPAA, M.H., NAKAJIMA, H.O., and FIELD, L.J. (2003). Physiological coupling of donor and host cardiomyocytes after cellular transplantation. Circ. Res. 92, 1217-1224.

SCHMALBRUCH, H. (1982). Skeletal muscle fibers of newborn rats are coupled by gap junctions. Dev. Biol. 91, 485-490.

SOONPAA, M.H., and FIELD, L.J. (1998). Survey of studies examining mammalian cardiomyocyte DNA synthesis. Circ. Res. 83, 15-26.
SUZUKI, K., BRAND, N.J., ALLEN, S., KHAN, M.A., FARRELL, A.O., MURTUZA, B., OAKLEY, R.E., and YACOUB, M.H. (2001). Overexpression of connexin 43 in skeletal myoblasts: Relevance to cell transplantation to the heart. J. Thorac. Cardiovasc. Surg. 122, $\underline{759-766 .}$

Address reprint requests to:

Dr. Hans Reinecke Department of Pathology

University of Washington Box 357470, HSB, Room D-514

Seattle, WA 98195-7470

E-mail: hreineck@u.washington.edu

Received for publication October 24, 2003; accepted after revision May 5, 2004.

Published online: May 24, 2004. 